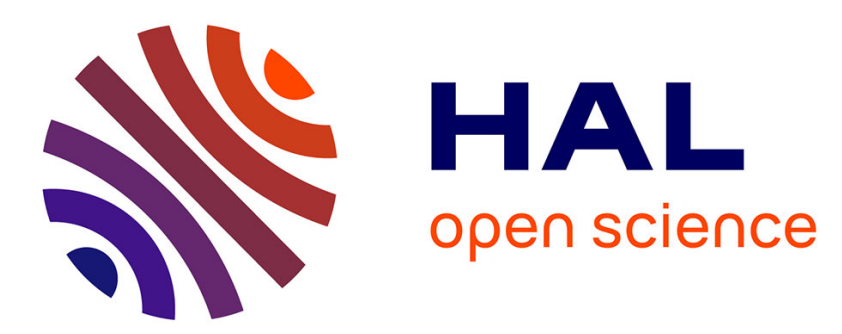

\title{
Local definition for surface-based geometrical modulus and use in automated casting cooling determination
}

\author{
Jean-Marc Auger
}

\section{To cite this version:}

Jean-Marc Auger. Local definition for surface-based geometrical modulus and use in automated casting cooling determination. International Journal of Cast Metals Research, The, 2017, 30 (6), pp.322 à 336. 10.1080/13640461.2017.1312737 . hal-01509892

\section{HAL Id: hal-01509892 \\ https://hal.science/hal-01509892}

Submitted on 4 May 2017

HAL is a multi-disciplinary open access archive for the deposit and dissemination of scientific research documents, whether they are published or not. The documents may come from teaching and research institutions in France or abroad, or from public or private research centers.
L'archive ouverte pluridisciplinaire HAL, est destinée au dépôt et à la diffusion de documents scientifiques de niveau recherche, publiés ou non, émanant des établissements d'enseignement et de recherche français ou étrangers, des laboratoires publics ou privés. 


\title{
Local definition for surface-based geometrical modulus and use in automated
}

\author{
casting cooling determination
}

\author{
Corresponding author
}

\author{
Auger Jean-Marc \\ École des Mines de Saint-Étienne, SPIN, CNRS 5307, LGF \\ 158 Cours Fauriel F-42023 Saint-Étienne, France \\ $+33477499735$ \\ jean-marc.auger@emse.fr
}

\begin{abstract}
The notion of geometrical modulus is widely used in foundry to approximate castings cooling through Chvorinov's rule. An extension to its definition at a local scale has been devised, which can be used to determine a cooling map of the surface of an arbitrary casting. An algorithm using simple tessellated surfaces (.stl) files as data entry has been tested, the results showing good agreement with theoretical values for simple casting shapes. Experiments have confirmed the potential of this method to automatically and quickly (a few seconds to minutes) map the cooling of complex parts, despite some limitations mostly linked to the initial discretization of the surface. Potential applications would be automated hot spots location, as well as determination of local cooling times and solidification directions.
\end{abstract}

Keywords: computer assisted design; foundry; cooling; geometrical modulus; Voronoï cell

\section{Introduction and context:}

Mould and casting designing is a centuries old problem. Experiments have led to some semiempirical rules which give accurate results in simple cases, at the cost of a long and difficult to automate process. Long designing times are not generally a concern, since moulds are even more time consuming to produce in industrial conditions, making experimental tests the critical step of the whole process. However, recent improvements in casting technologies (rapid prototyping in particular), have allowed a significant decrease in both time and cost of mould production. The subject of a quicker computer assisted casting designing is thus becoming increasingly relevant to industrial foundry production.

Two main types of approaches are currently used in order to design castings:

- traditional foundry, which is based on Chvorinov law (linking the solidification time of a casting with purely geometrical considerations, summarized by the geometrical modulus), coupled with semi-empirical rules in order to streamline the casting geometry. This method benefits from its simplicity (calculations limited to basic mathematical operations), minimal necessary data (just the casting geometry), and pre-existing foundry rules (risers dimensions for example - [1]). Its main limitations are linked to three characteristics: its global nature (for example, it will consider the solidification of a cube shaped casting as one entity, completely ignoring the progressive cooling from surface to core); dependence on experience and user know-how (especially to partition the casting into elements having uniform cooling); and an output limited to the relative cooling of the different parts of the casting.

These methods were designed from start to optimize the foundry process and avoid defects.

- $\quad$ numerical simulations, mainly finite elements and finite volumes methods. These tools can 
accurately describe both filling and solidification, using well known physical laws and models (thermodynamics, fluid mechanics, thermal diffusion...) applied to local elementary volumes, and calculating interaction between them. The main advantage of these methods is the access to physical data following the simulation [2], including evolution of the system with time and derived physical information (grain size for example). Limits of this kind of approach are long computation times (which can be several hours or days), and heavy reliance on the accuracy of base data not easily obtained (phase diagram of alloys, heat diffusion coefficients...).

This approach was mostly designed to predict the result of a given foundry process, thus the need to go through several design modifications/simulations cycles.

A few other methods, such as those based on largest included sphere [3, 4], exemplified in the medial surface/axis transformation [5, 6], or constructions in relation to Chvorinov rule ([7] for example), try to find a middle ground between accuracy and computation speed. However, their theoretical bases are very often debatable, and the results obtained not compatible with existing foundry rules, thus limiting their usefulness in industrial context.

As a conclusion, there is currently no easy and quick method to qualitatively predict the solidification of a casting that does not necessitate a great deal of experience in foundry beforehand, or time intensive computations. On one hand, simulation allows precise results, but those are not directly usable for designing the casting, and too long to obtain to be readily used in casting conception process. On the other hand, the traditional approach gives fast and effective answers for casting design (optimal risers or cooling blocks size and position can be directly deduced from geometric modulus for example), but cannot be automated, due the need of "intuitive" decisions concerning the partitioning of the casting into thermally significant entities.

This article will first detail a local definition for geometrical modulus based on physical considerations, then develop a discrete approach using Voronoï cells to approximate it (formal convergence being demonstrated in the case of plate shape). Considerations about numerical implementation will be discussed, leading to a working algorithm for random casting shapes. Finally, computations results on simple and complex shapes will be shown and analyzed, highlighting limitations of the process and ways to optimize it.

\section{Theoretical approach}

\section{Preliminary theoretical considerations:}

The main idea of the method described in this article, is to define a local surface value for geometrical modulus, then integrate this result to differentiate thermally significant parts of the casting. This is the opposite of the traditional foundry approach, where the casting is first partitioned into elementary entities, each being intuitively thermally homogeneous, then the modulus calculated. The new method has the advantage to avoid the prerequisite knowledge of foundry rules for partitioning a casting.

In order to have a physical significance, the mathematical definition of local modulus must follow the same properties and geometry as the phenomenon described, namely thermal diffusion. It must also be based on the same hypotheses that link the initial definition of global geometrical modulus to the solidification time of a casting. This last imperative is necessary, on one hand to maintain some consistency between the two definition scales of the modulus (global and local), and on the other hand to be able to directly use the resulting local modulus with existing foundry rules.

These essential hypotheses will be determined below using various considerations, first about geometrical modulus and its link with cooling, and then about thermal diffusion in a casting.

Chvorinov initial observations [8] have led to the discovery of a relationship between cooling duration and casting geometry:

$$
t_{s}=k \cdot M^{2}
$$

With $t_{s}$ the cooling duration to solidify the liquid metal, $\mathrm{k}$ a coefficient dependent on mould conditions and alloy composition (for example, see [9] and [10] for further details and value determination), and $M$ the geometrical modulus, defined by the ratio between the volume of the casting and its cooling surface.

Demonstration of this relationship can be obtained by theoretical considerations using the case of plate shaped castings. As such, Chvorinov's rule is based on the hypotheses of uniaxial thermal diffusion, uniformity of thermal diffusion properties on the mould surface and uniformity of initial temperature. Further studies [11] have somewhat refined and expanded this result to determine the solidification time as: 


$$
t_{s}=k \cdot M^{n}
$$

with $\mathrm{n}$ a corrected exponent comprised between 1.5 and 2, that takes casting curvature (and thus tridimensional thermal diffusion) into account.

Concerning the geometry of thermal diffusion, owing to homogeneous thermal properties at the inner surface of the mould and uniformity of temperature, thermal flux is initially normal to the surface of the casting. However, due to the different cooling rates existing in the various casting parts, the thermal flux direction changes with time. In the framework of Chvorinov's rule, the cooling duration is linked to the initial conditions (more precisely, the shape of liquid metal before cooling) of the casting, making it a first order (in time) model for thermal diffusion. Consequently, a consistent hypothesis would be that the thermal flux retains its initial orientation, normal to the surface.

\section{Properties of elementary Voronoï cells generated by the surface of a casting:}

We consider a point on the surface of the casting to be designed. The corresponding Voronoï cell generated by all the other points constituting the surface, and further limited by the outer surface of the casting, will have the following properties:

- points inside the cell volume are (by construction), the nearest to the generating point; consequently, points inside the volume of the cell are also the nearest to the elementary outer surface (this surface will be called the umbrella of the cell) around the generating point

- $\quad$ the convexity of Voronoï cell inner surfaces (surfaces excluding the umbrella) ensures that thermal flux normal to the umbrella does not cross another cell

These elements ensure that the thermal energy contained within the elementary Voronoï cell will (under the assumption of a thermal flux normal to the initial cooling surface) diffuse exclusively through the cooling surface that is the umbrella. The elementary Voronoï cell is a meaningful thermal entity that could be considered isolated from the other cells in the framework of the previously detailed hypotheses. It is thus possible to define a local value of geometrical modulus by calculating the ratio between the volume and the umbrella surface (cooling surface) of a Voronoï cell, which is generated by the surface of the casting around any of its points. While satisfying from a theoretical point of view, this definition of local modulus is of little practical use, as calculations would have to be made for an infinite number of points on the surface. It is therefore interesting to study a discrete approximation of this local modulus, using a similar construction in order to keep compatible properties.

\section{Elementary Voronoï cell approximation using Delaunay triangulation of surface:}

A typical discrete approximation of surfaces with triangular facets will be used as a basis for the approximation of elementary Voronoï cells. In that case there is a finite number of vertices used to generate the cells, with the following properties:

- lateral facets are normal to the vectors linking the generating vertex with nearby vertices, and thus are normal to the facets defining the umbrella of the cell. The construction of the discrete Voronoï cell is compatible with the hypothesis of a thermal flux always normal to the casting surface.

- the second hypothesis, stating that each point inside a cell is nearer to the cell umbrella than any other outer surface, is obviously not verified in the case of triangular tessellation of the surface. However, it is possible to demonstrate the convergence of the approximation on the theoretical elementary cell. Such demonstration will be detailed in the simple case of 3 dimensions infinite plate.

\section{Convergence (3D infinite plate) :}

Theorem 1: the Voronoï cell wall defined by a vertex and a plane is a paraboloid of revolution

Due to the rotational symmetry of the problem, we first consider two parallel lines separated by a distance of e. A generating vertex $G$ is situated on the upper line. Coordinates are given in the natural orthogonal coordinate system centered on $G(G, x$, $y)$. The equation of the line (potentially) defining the boundary of the Voronoï cell generated by $\mathrm{G}$ and a given point of the lower line (coordinates $(X,-e))$ is:

$$
X . x+e . y-\frac{\left(e^{2}+X^{2}\right)}{2}=0 ; y \in\left[\frac{-e}{2} ; 0\right]
$$

The inside surface of the Voronoï cell defined by the generating vertex $G$ and each point of the lower line can be expressed by the following system:

$$
x \leq \min _{X \geq 0}\left\{\frac{-e \cdot y}{X}+\frac{\left(e^{2}+X^{2}\right)}{2 \cdot X}\right\}
$$




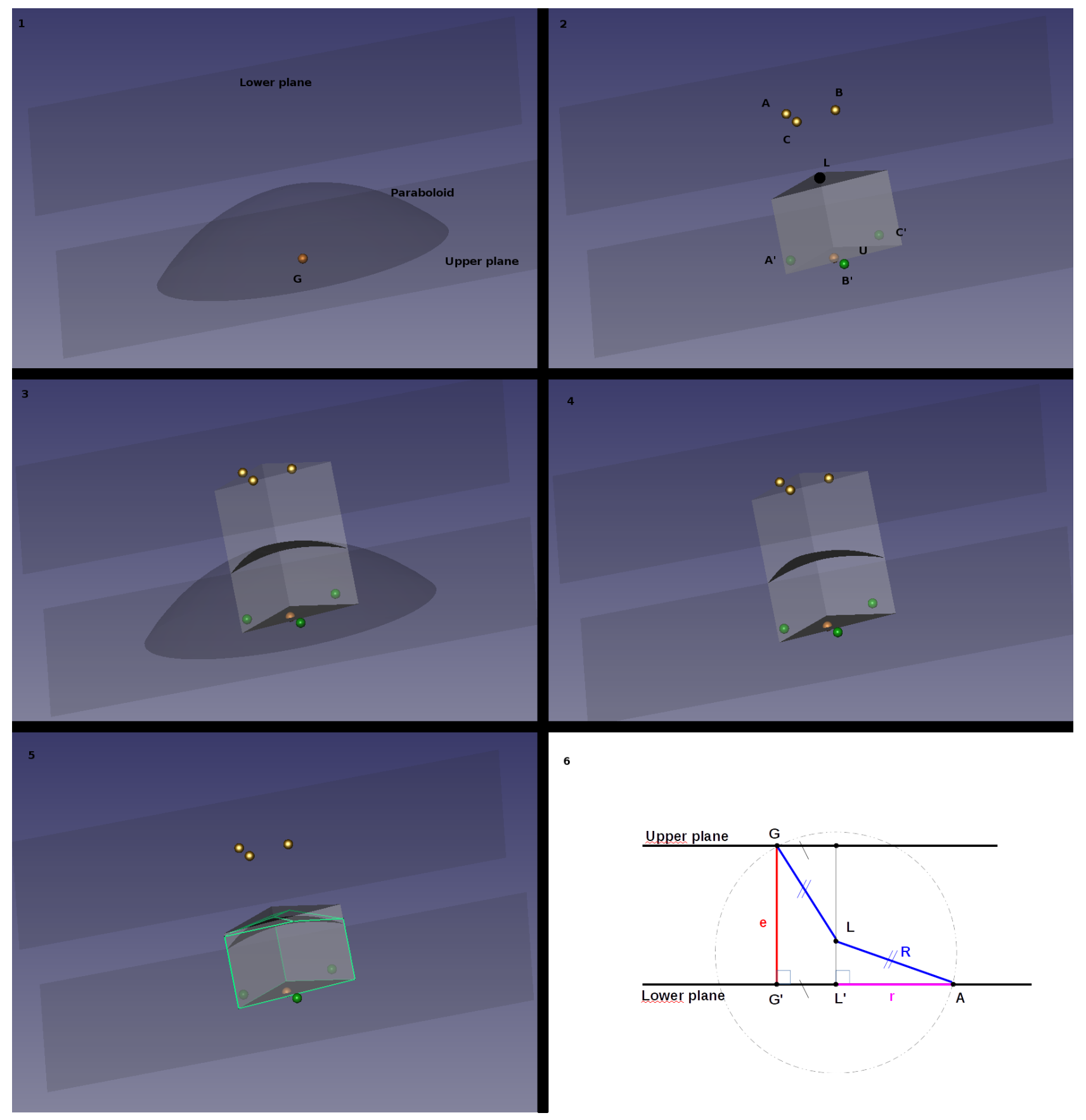

Figure 1: Construction of bounds for Voronoï cell surface in 3D case (1- paraboloid delimiting points nearer to $G$ than any point of the lower plane, 2-upper bound of Voronoï cell defined by $G$ and its nearest neighbours belonging to the mesh A, B, C, A', B' and C', 3-construction of lower bound by intersection of the right prism of base $U$ and the paraboloid, 4- prism and lower bound, 5- Voronoï cell and its upper and lower bounds, 6- geometrical construction used to determine the distance between highest and lowest points of Voronoï cell)

$$
x \geq \max _{X \leq 0}\left\{\frac{-e \cdot y}{X}+\frac{\left(e^{2}+X^{2}\right)}{2 \cdot X}\right\}
$$

Simple derivations with respect to $X$ lead to the equation of the Voronoï cell boundary: 


$$
y=\frac{\left(X^{2}-e^{2}\right)}{2 . e}=0
$$

which, considering the rotational symmetry of the problem, defines a paraboloid of revolution (see Fig. 1-1). As a side, but interesting, note, the volume thus enclosed is the smallest possible for a Voronoi cell generated by $G$ and any number of points belonging to the plane. The intersection between the paraboloid and a parallel plane going through $G$ is the circle of radius e centered on $\mathrm{G}$.

From this point on, an infinite plate of thickness e is considered. Its outer surface consists of two parallel planes, each discretized with a density of points per surface unit of respectively $n_{\text {sup }}$ and $n_{\text {inf }}$, for the upper and lower plane. A generating vertex $G$ is situated on the upper plane, with an umbrella $U(2 D$ Voronoï cell defined by $G$ and its nearest neighbours on the upper plane) of surface $S$.

Theorem 2: upper boundary of the distance between generating vertex and lowest point of the Voronoï cell

We consider the generating vertex $G$ and $A, B, C$ its three nearest non-aligned vertices on the lower plane (thus $G^{\prime}$, orthogonal projection of $G$ on the lower plane is located inside the ABC triangle). By construction of the Voronoï cell, its lowest point $L$ is equidistant from $\mathrm{G}, \mathrm{A}, \mathrm{B}$ and $\mathrm{C}$.

$$
R=A L=B L=C L=G L
$$

$L^{\prime}$, orthogonal projection of $L$ on the lower plane, is thus the center of the circumscribed circle of $A B C$ triangle:

$$
r=A L^{\prime}=B L^{\prime}=C L^{\prime}=G L^{\prime}
$$

Simple considerations on ALL' and GG'L' right angled triangles (see Fig. 1-6) give :

$$
R^{2}=L L^{\prime 2}+r^{2}
$$

and

$$
R^{2}=\left(L L^{\prime}+e\right)^{2}+G^{\prime} L^{\prime 2}
$$

if $L$ is under the lower plane, or

$$
R^{2}=\left(L L^{\prime}-e\right)^{2}+G^{\prime} L^{\prime 2}
$$

if $L$ is between upper and lower planes.

Combining equations 9,10 and 11 gives:

$$
\begin{aligned}
R^{2}= & \frac{e^{2}}{4}+r^{2}+\frac{1}{4} \cdot\left(\frac{r^{2}-G^{\prime} L^{\prime 2}}{e}\right)^{2}-\frac{r^{2}-G^{\prime} L^{\prime 2}}{2} \\
& r^{2} \geq G^{\prime} L^{\prime 2} \geq 0
\end{aligned}
$$

and finally

$$
R \leq \sqrt{\frac{e^{2}}{4}+r^{2}+\frac{r^{4}}{4 \cdot e^{2}}}
$$

giving a higher boundary of the Voronoï cell distance between its highest and lowest points.

Theorem 3: convergence of discrete geometrical modulus when discretization length decreases

According to [12] and [13], the maximum distance between linked vertices in a Delaunay triangulation $I_{\max }$, is related to the vertex density $\mathrm{n}$ by:

$$
I_{\max }=O\left(\sqrt{\frac{\log (n)}{n}}\right)
$$

with a limit of 0 as density increases.

The area $S$ of the umbrella $U$ around vertex $G$ decreases when density $n_{\text {sup }}$ increases. With a high enough vertex density $\left(n_{\text {sup }}>N_{\text {sup }}\right)$, the umbrella is thus included inside the circle of radius e centered on $\mathrm{G}$.

By construction ( $G$ and its neighbours being coplanar), the Voronoï cell generated by $G$ is included in the right prism of base $U$. A lower boundary of the cell volume can then be obtained by the intersection of the prism with the paraboloid defined in Theorem 1 (as visible in Fig. 1 -3). When $n_{\text {sup }}>N_{\text {sup }}$, the boundary volume can be separated in two parts: first, the volume where the prism is completely included in the paraboloid; then, the rest. Only the former will be considered for the definition of the lower boundary of the Voronoï cell volume. Considering $I_{\max }(U)$ the upper boundary of the distance between $G$ and any point belonging to $U$, and using equation 6 , the height $h$ of the included prism is given by:

$$
h=\frac{e}{2}-\frac{l_{\max }(U)}{2 . e}
$$


On the other hand, the volume of the cell can be easily upper bound by the product of the umbrella surface by the maximum height of the Voronoï cell as defined in theorem 2. These elements lead to the upper and lower bounds of the Voronoï cell volume (see Fig. 1-2 for the upper boundary, Fig.1-4 for the lower one, and Fig.1-5 for the cell constructed between its boundaries).

$$
\begin{aligned}
& S \cdot\left(\frac{e}{2}-\frac{l_{\max }(U)}{2 . e}\right) \leq V_{\text {cell }} \leq S \cdot \sqrt{\frac{e^{2}}{4}+r^{2}+\frac{r^{4}}{4 . e^{2}}} \\
& r \leq l_{\text {max }}(\inf )=O\left(\sqrt{\frac{\log \left(n_{\text {inf }}\right)}{n_{\text {inf }}}}\right) \\
& l_{\text {max }}(U)=O\left(\sqrt{\frac{\log \left(n_{\text {sup }}\right)}{n_{\text {sup }}}}\right)
\end{aligned}
$$

$$
S \cdot\left(\frac{\left.\frac{e}{2}-O\left(\sqrt{\frac{\log \left(n_{\text {sup }}\right)}{n_{\text {sup }}}}\right)\right)}{V_{\text {cell }} \leq S \cdot \sqrt{\frac{e^{2}}{4}+O\left(\sqrt{\frac{\log \left(n_{\text {inf }}\right)}{n_{\text {inf }}}}\right)}}\right.
$$

thus providing upper and lower bounds for the geometrical modulus of the cell, and proving its convergence when both upper and lower planes vertices density increases:

$$
\frac{e}{2}-O\left(\sqrt{\frac{\log \left(n_{\text {sup }}\right)}{n_{\text {sup }}}}\right) \leq M_{\text {cell }} \leq \sqrt{\frac{e^{2}}{4}+O\left(\sqrt{\frac{\log \left(n_{\text {inf }}\right)}{n_{\text {inf }}}}\right)}
$$

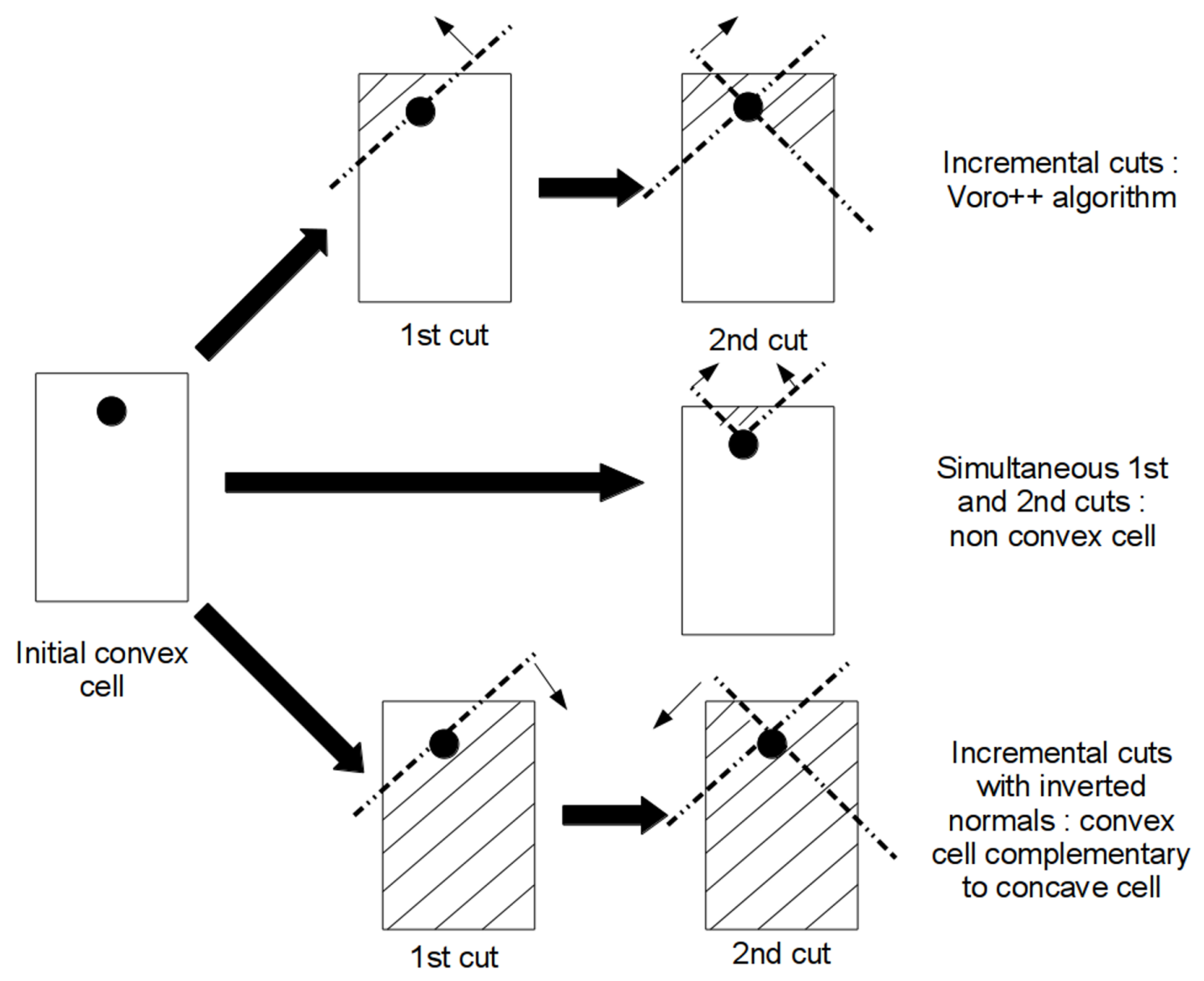

Figure 2: Principle of computations for non-convex Voronoï cells 


\section{Numerical implementation details:}

The input file uses the .stl ASCII format: triangular facets are defined by the coordinates of three vertices and a normal unit vector, indicating the outside of the solid. This choice was made in order to have a widespread and simple to handle input format.

The core of the Voronoï cells calculations is done using the Voro++ C++ library [14]. According to [15], this library allows simple and rather quick computations, the main limitation being its inability to handle concavities in Voronoï cells.

\section{Summary overview of Voro++ computation scheme [15]:}

For each vertex, the to-be calculated Voronoï cell is first initialized as a global parallelepipedic container. Series of walls (planes) are then applied sequentially to the cell, cutting it and removing parts of its volume. Walls can have two differing origins:

- perpendicular bisector planes between the current vertex and its neighbours, that correspond to the standard definition of a Voronoï cell. These planes will be named inner walls.

- planes that approximate the outer shell of the solid studied. In the case of a surface that is already tessellated, such as stl files, there is no error risk between Voronoï calculations and initial data. These planes will be defined as outer walls.

As a side note, the incremental use of walls in this algorithm accounts for the impossibility of nonconvex cells. More precisely : the outside of purely convex Voronoï cells is defined, unequivocally, by the union in the mathematical sense of all outer halfspaces (area of space that is on the outer side of a wall) - see first line of Fig. 2. The case of nonconvex cells is more complex, as concavity exists only locally. Outer half-spaces should be divided between those linked to planes delimiting a convex solid (convex outer half-spaces) and those that locally define a concavity (concave outer halfspaces); the outside of the Voronoï cell is then the union of all convex outer half-spaces with all local intersections of concave outer half-spaces. As such, the definition of local concavities requires to simultaneously cut the initial solid by more than one wall or plane (see second line of Fig. 2), which is not possible in the Voro++ framework. This problem could be solved by a decomposition of the solid in convex parts. However, according to existing work [16], there is no simple way to implement this decomposition in the general case of an arbitrary geometry.

A workaround, however, has been devised, owing to several facts:

- Voronoï cells defined only by interaction between points (without any outer wall) are always convex (general property of Voronoï cells). Concavity can thus only happen on parts of the cell defined by the umbrella.

- The tessellation of the outer surface means that vertices are always part of the surface.

- The tessellation of the outer surface with triangles leads to an interesting property: all concavities (which always exist as edges of the mesh in this case) are in direct contact with vertices.

With these conditions, it is possible to simply compute non-convex cells by following a two-steps procedure: first, determining an enveloping convex cell, defined by the inner walls and the maximum subset of outer walls that defines a convex outer surface; then determining the complimentary convex cell, defined by the inner walls and the subset of outer walls that defines a concave outer surface, with inverted normals (see last line Fig. 2). Each of the enveloping and complimentary cell is convex, and can thus be directly computed by Voro++ library. The final non convex Voronoï cell (limited by the full complement of outer walls) is then easily obtained as the difference between the enveloping and complimentary cells (see Fig. 3 ).

\section{Detailed steps of the geometrical modulus computation (summarized in Fig. 4 :}

- Pre-treatment of stl file:

For each vertex, the facets comprising its umbrella are separated into two categories: "concave" facets and "convex" facets. For each facet of the umbrella, the position of all other vertex belonging to the same umbrella relative to the exterior normal of the facet is tested; if all points are "below" the facet plane, the facet will be considered convex; if at least one point is "above" the plane, the facet is considered concave, relatively to the current vertex (a given facet belongs to three different umbrellas, and can 


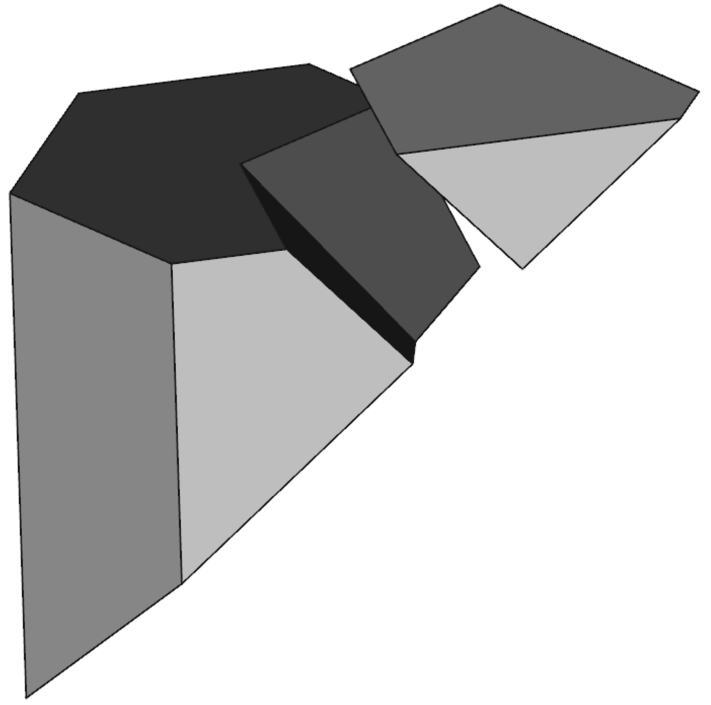

Figure 3: Example of 3D Voronoï non convex cell (left), with complimentary cell (right). Cooling surface (umbrella) is visible in green. thus be categorized as convex relatively to some of of them, while being considered concave relatively to the others).

A list is then created, that contains the coordinates of the vertex at the origin of the umbrella, the coordinates of the normal to one facet belonging to the umbrella, and an indicator of the concavity associated with this facet as part of this umbrella.

- The vertices and facets (walls) list is applied to the Voro++ computation scheme twice: first, only taking "convex" facets for each vertex into account (thus generating enveloping convex cells of the real Voronoï cells); the second time, all facets are used in the calculation, but the normals of "concave" facets are inverted (leading to the complementary cells to the real ones).

Each Voronoï calculation generates two different outputs : a list of cells with their associated volumes, and a list of cells with their associated facets surfaces. The surfaces (polygons), are identified by a relative integer each, with the polygons

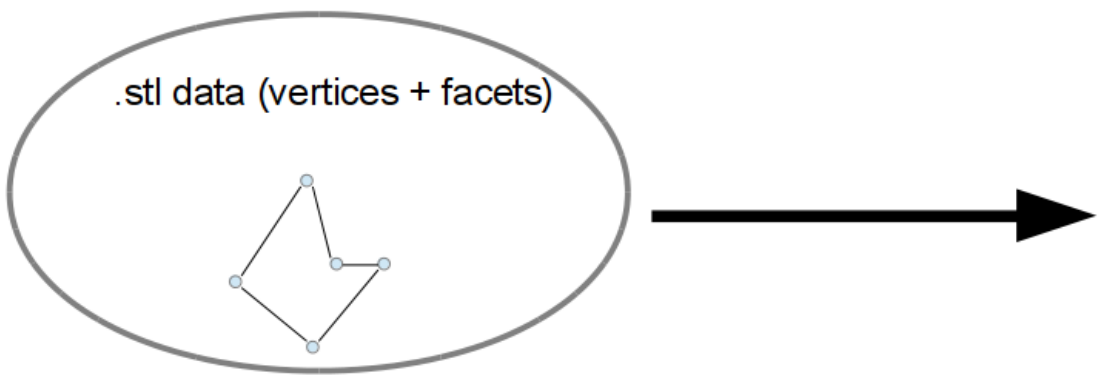

1-Pre-treatment
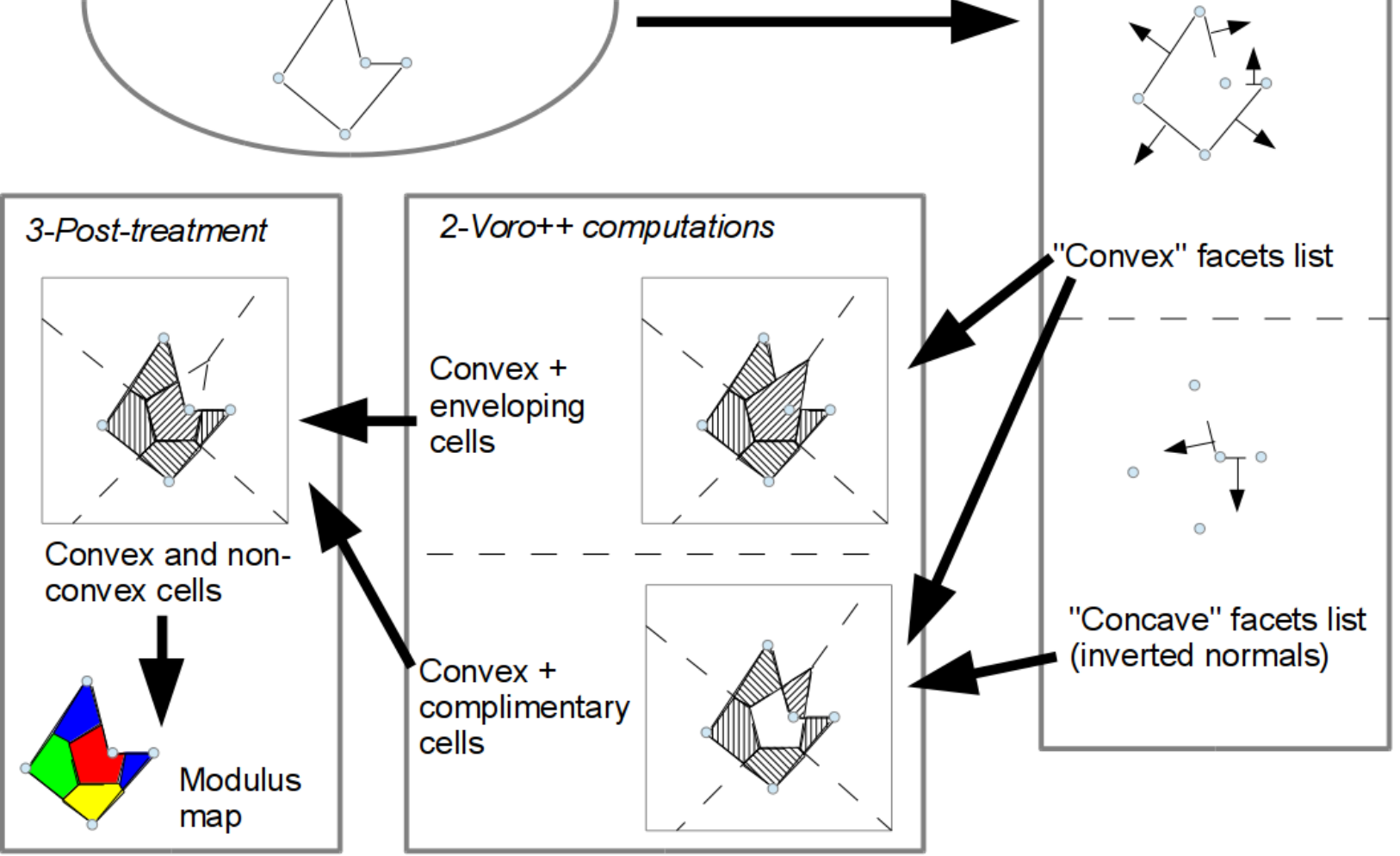

Figure 4: Steps of geometrical modulus computation algorithm 
corresponding to contacts between cells having positive identifiers, and the outer walls (cooling surfaces) being identified by negative values.

- Post-treatment: for each cell, the results of the two computations are combined, in order to determine both its volume and cooling surface.

Cell volume is easily obtained by the difference between the volumes of enveloping cell and complimentary cell:

$$
V_{\text {voronoi }}=V_{\text {convex }}-V_{\text {concave }}
$$

Cooling surface, however, is more difficult to calculate, as it requires identifying common surfaces from enveloping and complimentary cells:

$$
S_{\text {voronoi }}=\left(S_{\text {convex }} \cup S_{\text {concave }}\right) /\left(S_{\text {convex }} \cap S_{\text {concave }}\right)
$$

First, the global cooling surface is initialized as the sum of cooling surfaces of the enveloping convex cell. A comparison of cooling surfaces between enveloping and complimentary cells is then carried out; if a cooling surface belongs to both computed cells, it is subtracted from the global surface value; in the other case, it is added to the global surface value.

Voronoï cell geometrical modulus is then deduced by a simple ratio between the two previously computed values. Results are exported in simple text file containing the coordinates of each generating vertex, with the associated modulus. Visualization of modulus mapping can then easily be carried out using point cloud analysis software.

This algorithm presents some important limitations:

- The vertex that generates a given Voronoï cell is not always at the gravity center of its umbrella, especially in the case of an irregular mesh or modulus gradient. This can lead to small errors if the computed geometrical modulus is considered a property of the generating vertex alone. A proper view would be to associate the modulus value of a Voronoï cell to its whole umbrella, rather than to its generating vertex. As a direct consequence, accurate modulus gradient determination on the surface of the mesh necessitates additional calculations.

- An excessively fine meshing of the surface can lead to considerable errors in the modulus value. This is due to the fixed precision of the computations, which becomes proportionately more important when the cooling surface and volume of the Voronoï cell decrease.

\section{Experimental results and interpretations:}

\section{Elementary shapes}

A few basic geometric shapes have been tested, as a means to evaluate the effective accuracy of the algorithm in simple situations: homogeneously thick plates, cylinders and wedges.

In order to assess the precision of the algorithm in the case of uniform cooling, the program was run on simple parallelepiped $20 \times 20 \times 2 \mathrm{~mm}$ plates, with discretization lengths ranging from $0.5 \mathrm{~mm}$ to $2 \mathrm{~mm}$ (by $0.1 \mathrm{~mm}$ steps), $2.5 \mathrm{~mm}$ and $3 \mathrm{~mm}$. Neglecting the increased cooling near the edges, the theoretical value of geometrical modulus in the center of the plate (more than $1 \mathrm{~mm}$ from the edges) should be $\mathrm{M}=1 \mathrm{~mm}$. Calculations give the right value, with no dispersion (standard deviation is 0). This "perfect" result, however, can be explained by the tessellation of the shape: since the two largest faces of the plate are symmetric, their triangular tessellations are perfectly identical, and their vertices superimposed when looking from a normal direction. All the calculated Voronoï cells are then limited by the plane separating the plate in the middle.

A way to avoid this effect is to use truncated right circular cones (frustums) instead of plates: since the tessellation is generated from the edges of the shape, varying apertures induce small variations in discretization lengths, which create shifts of the vertices between the two opposite faces. The dimensions of the frustums (thickness $2 \mathrm{~mm}$ ) studied are summarized in Table 1, along with the effective discretization lengths (calculated as the square root of the ratio between surface area and number of vertices) for each face. The following general observations can be carried out on the results:

- The maximum error of calculated geometrical modulus in each of the frustums is very small: less than $1 \%$ in all cases (see Fig. 5). This value is far less than the traditional modulus threshold of $20 \%$ used to determine significant cooling difference in a part (equivalent to $50 \%$ more or less cooling time, standard definition commonly used in traditional foundry). 

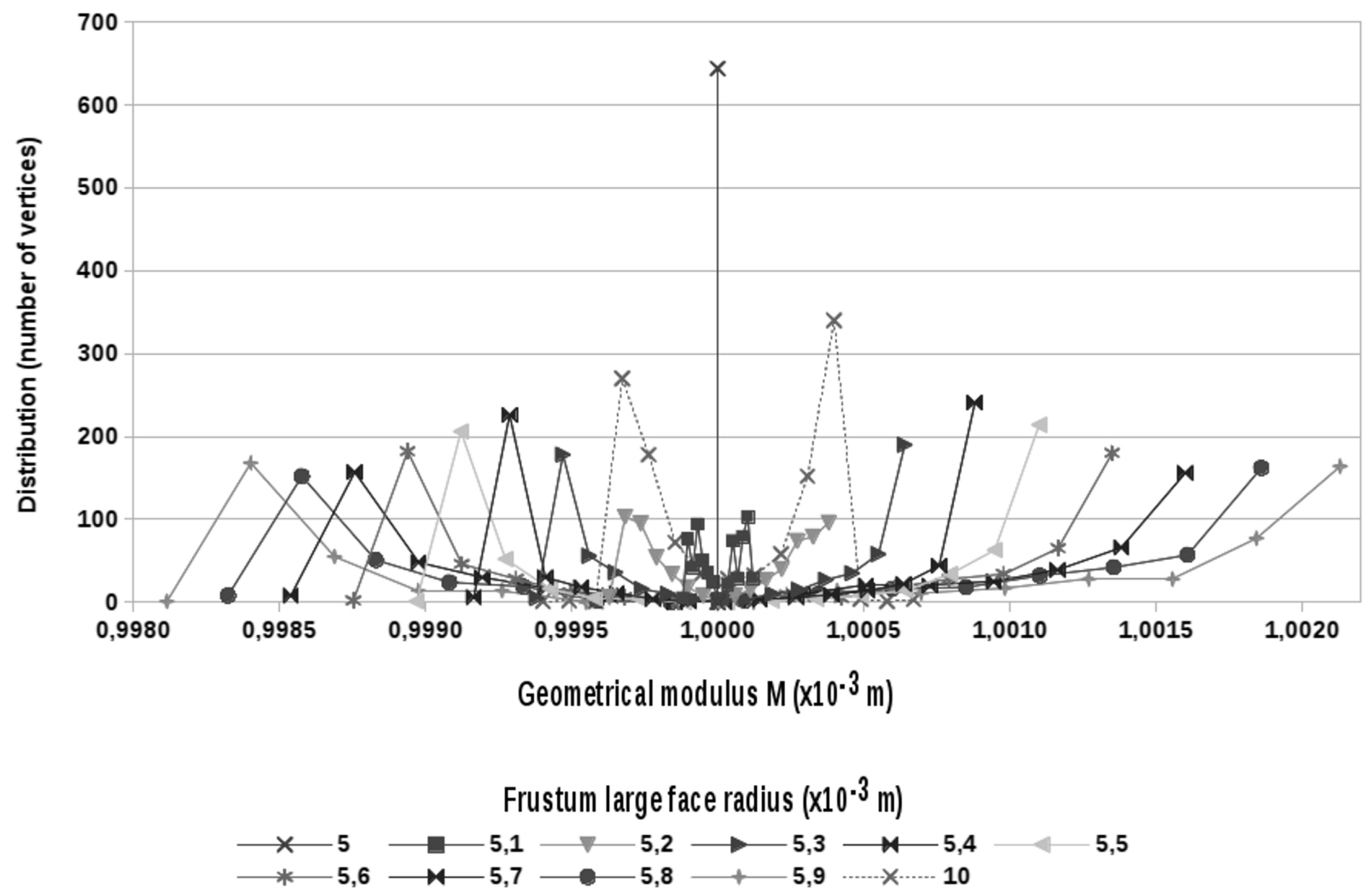

Figure 5: Geometrical modulus $M$ distribution for varying large face radius frustums (discretization length $0.5 \mathrm{~mm}$ )

- Modulus repartition (taking into account the two opposing faces) is centered on the theoretical modulus, with a predominance of the most extreme values (see Fig. 5).

- The series of tests with large frustum face radius ranging from 5 to $5.9 \mathrm{~mm}$ shows increasing (resp. decreasing) maximum (resp. minimum) values as the radius increases. This effect can be explained by a progressive misalignment of vertices on the two opposing faces: when the radius is $5 \mathrm{~mm}$, the meshes are perfectly symmetric (as in the parallelepiped shape), and computations yield only theoretical modulus value; as the radius is increased, vertices drift farther away, leading to errors. This explanation is further confirmed by the nonrandom nature of modulus repartition on the faces when observed with a dilated color scale, in order to evidence small variations (see Fig. 6), which follows the pattern of mismatched meshes.

- For a given frustum shape, an increase of the discretization length from $0,5 \mathrm{~mm}$ to $0.7 \mathrm{~mm}$ leads to more extreme values of the computed geometrical modulus. This result seems in agreement with the convergence of calculated modulus with diminishing discretization length. However, the former observations concerning vertices alignment cast some doubt about the validity of this experimental confirmation, as it cannot be carried out for every mesh configuration imaginable.

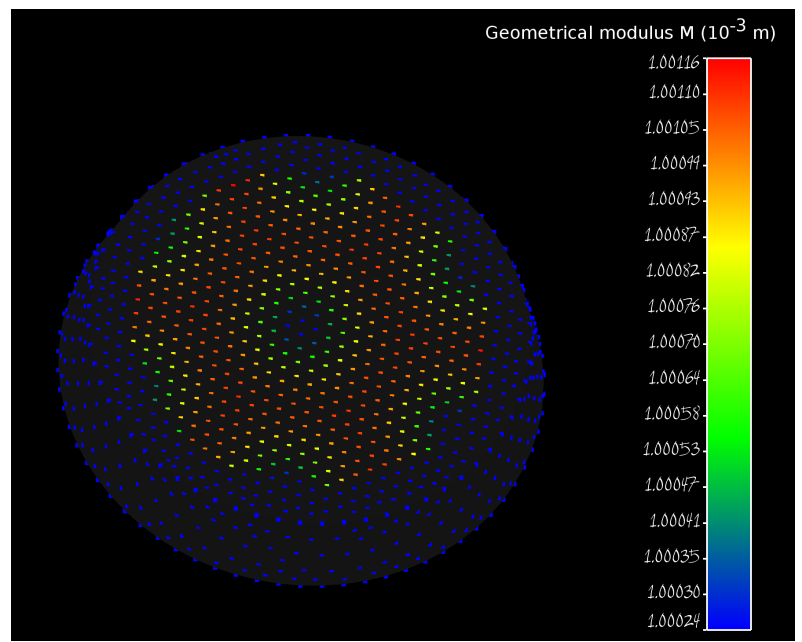

Figure 6: Geometrical modulus repartition on the face of a frustum (discretization length 0.5 $\mathrm{mm}$ - small radius $5 \mathrm{~mm}$ - large radius $5.5 \mathrm{~mm}$ ) 
As a summary of geometrical modulus computations on plate-like shapes, two main characteristics can be evidenced : first, a good agreement (less than $1 \%)$ of the calculated modulus with its theoretical value (result to be taken cautiously due to the other characteristic described below); second, the foremost importance of vertices positions (especially alignment), which is difficult to control during mesh generation, and can completely conceal all other effects in the framework a finite number of experimental tests.

Cylinder shape was used to study the effect of a constant surface curvature combined with a set geometrical modulus. Cylinder radius is $2 \mathrm{~mm}$, and its length is $10 \mathrm{~mm}$. The area of interest is located at a distance of at least $2 \mathrm{~mm}$ from extremities, in order to avoid the increased cooling at boundaries. With these parameters, the cylinder part studied can be considered equivalent to a section of an infinite, 2 $\mathrm{mm}$ radius, cylinder, leading to an homogeneous theoretical modulus of $1 \mathrm{~mm}$ (half of cylinder radius). Varying discretization lengths were used, ranging from $0.2 \mathrm{~mm}$ to $1.5 \mathrm{~mm}$. Main results are summarized in Fig. 7, and further discussed below:

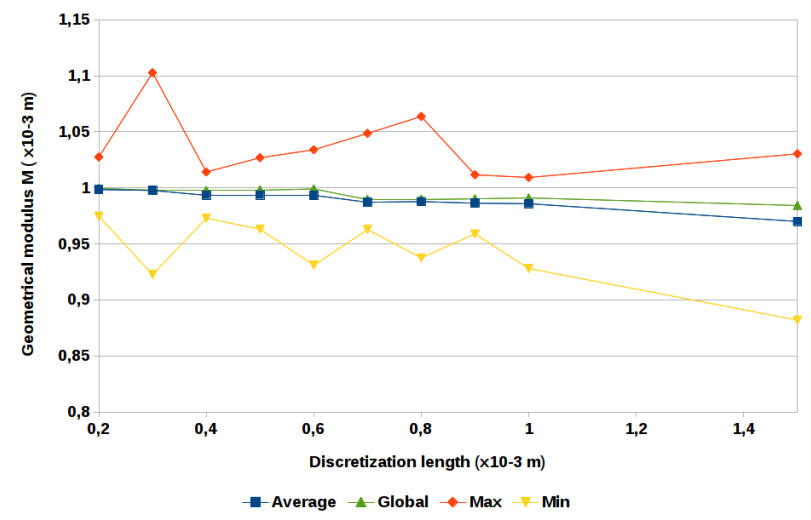

Figure 7: Geometrical modulus evolution with discretization length for cylinder shape (extreme, average and global values)

- The average geometrical modulus values are near the theoretical value, with increasing accuracy when discretization length decreases. Furthermore, the average geometrical modulus decreases with increasing discretization length. These effects are the result of two different phenomena: first, the shape tessellation, which means that the geometry analyzed is not exactly that of a cylinder; second, the errors associated with the geometrical modulus calculations through Voronoï cells. In order to isolate the consequences of each phenomenon, the discretized cylinders "global" theoretical modulus was estimated by the ratio of their true volume and surface (not taking the extreme closing disks into account). The word "global" here refers to the value of geometrical modulus if it was perfectly uniform in the geometry studied. Comparison between the global modulus and its average computed value (Fig. 7) shows that their evolutions with discretization length are similar (decreasing), but slightly less pronounced in the case of the global module (difference is less than $1.5 \%)$. This difference is probably due to an inadequate comparison, as the averaging of local module values does not take the weight (volume) of each Voronoï cell into account.

- $\quad$ The extreme values of geometrical modulus seen in the cylinder for each discretization length are less than $12 \%$ away from the average value. This means that the results accuracy is high enough to distinguish geometric entities with homogeneous cooling, as these can be defined by a continuous volume where the modulus shows less than $20 \%$ variations.

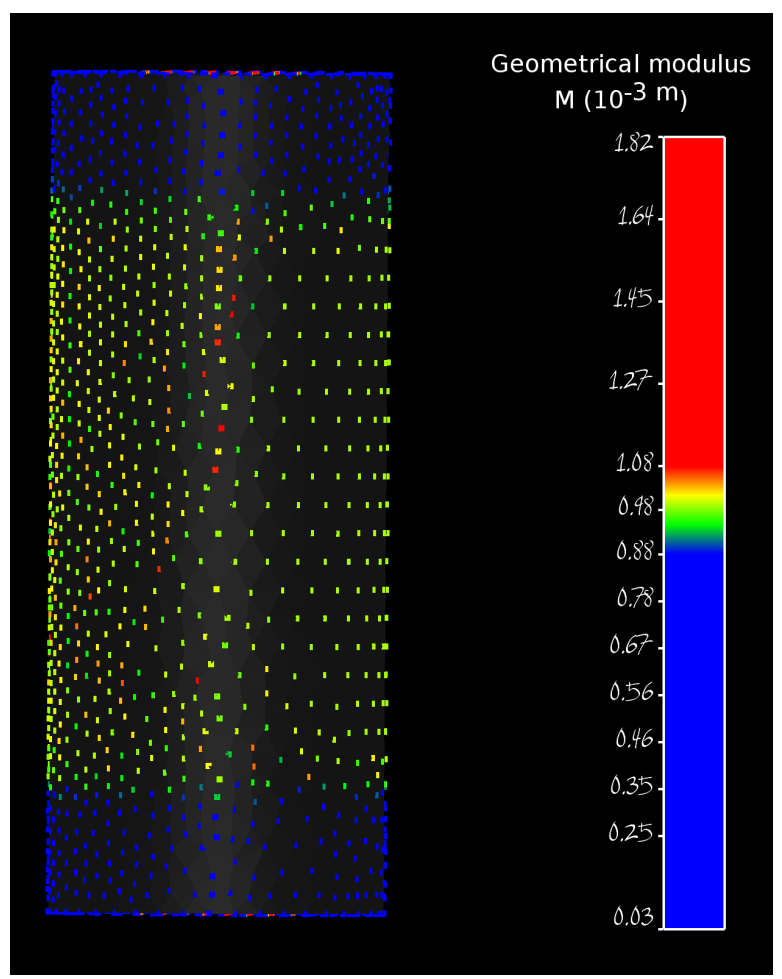

Figure 8: Link between geometrical modulus errors and mesh irregularity; center: high modulus errors with highly irregular mesh, right side: homogeneous modulus on regular mesh $(2 \mathrm{~mm}$ radius cylinder $-0.3 \mathrm{~mm}$ discretization length)

- Extreme modulus values evolution do not seem to correlate at all with discretization length. However, the vertices presenting extreme (whether high or low) modulus values are located in areas of the cylinder with a change of vertices 
density, as visible in central part of Fig 8 . Furthermore, high regularity of the mesh (visible by simple observation) correlates well with very low standard deviation and limited extreme values of the modulus, as seen in the case of 0.4 $\mathrm{mm}$ discretization length (see right part of Fig. 8). As a conclusion, and for cylinder shapes, mesh regularity is a first order factor controlling the accuracy of geometrical modulus computations, with discretization length being a second order parameter that determines the maximum potential amplitude for these errors, as is the case with plate shapes. Due to these two main effects, no influence of curvature has been evidenced. Another interesting consequence is that discretization errors on the shape $(1.5 \%$ maximum difference between global and theoretical modulus) are negligible compared to local effect of mesh irregularities $(1.5 \%$ to $10.5 \%$ difference between global and extreme modulus values).

Lastly, a wedge shape was the obvious choice to study the effect of geometrical modulus gradient. In order to increase the number of vertices analyzed by using symmetries, computations were carried out on a triangular right prism of height $10 \mathrm{~mm}$. Its base is an isosceles triangle with $10^{\circ}$ angles and a third side of length $20 \mathrm{~mm}$. Discretization lengths of 0.5 , $0.6,0.7$ and $1 \mathrm{~mm}$ were used. With this simple geometry, theoretical geometrical modulus can easily be expressed as a function of the distance $x$ to the edge of angle $\alpha$ (not taking into account other edge effects, as is the case for an infinite wedge):

$$
M(x)=x \cdot \tan \left(\frac{\alpha}{2}\right)
$$

Increased cooling near the other edges is neglected, by not taking the vertices located nearer than $2 \mathrm{~mm}$ from these edges into account for the following results:

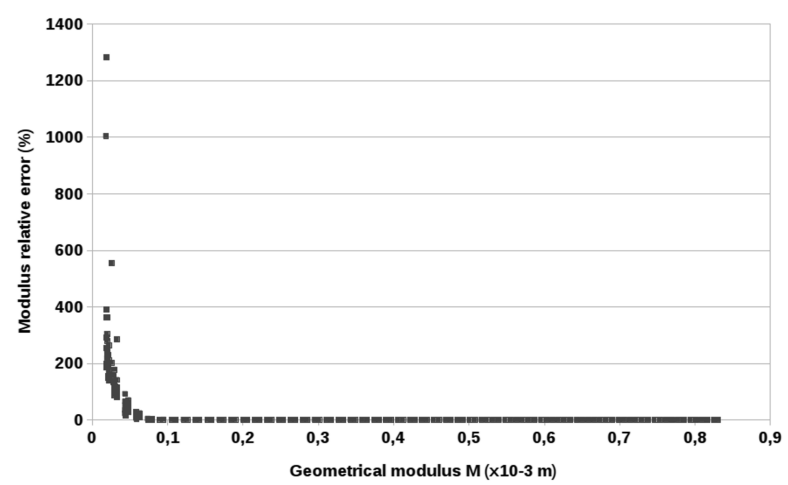

Figure 9: Relative error of computed geometrical modulus as a function of theoretical modulus (discretization length of $0.5 \mathrm{~mm}$ )
- First, for a set discretization length, a decrease of the geometrical modulus (which corresponds to a lower distance to the angle edge) leads to an increase of the maximum relative error of the computation (see Fig. 9). Experimental errors show an exponential evolution when the modulus decreases. As a consequence, it should be possible to define a minimum modulus below which the error becomes significant in a foundry context (more than $20 \%$ error). However, this experimental threshold shows no direct correlation with discretization length, and cannot be used to define an upper boundary for discretization in order to limit errors.

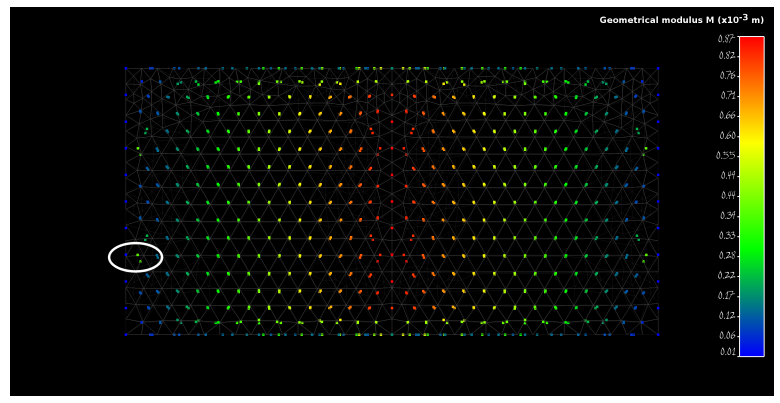

Figure 10: Correlation between high geometrical modulus error and meshes misalignment on a prism shape $(0.6 \mathrm{~mm}$ discretization length) - view from above

- $\quad$ On the other hand, for a given geometrical modulus, the maximum error on the computed value shows no obvious correlation with discretization length. This observation seems contradictory with a convergence of the calculated values with decreasing discretization lengths (synonymous to increasing vertices density). However, a local comparison of high and low error Voronoï cells yields the following results : low error cells correspond to aligned vertices on the planes constituting the wedge, whereas high error cells are located where meshes of the planes are dissimilar (see Fig. 10). In a way similar to the plate shape, the potential maximum error value for a given modulus is indeed determined by discretization length, but the degree to which this potential is realized in a given casting shape depends solely on vertices alignment

- Finally, errors appearing in low thickness locations can be especially high due to another effect, linked to the algorithm used. As visible in Fig. 11 [Figure 11 near here], which shows a representation of the Voronoï cells constituting the shape, some cells near the edge are elongating far outside of the solid volume (out- 
stepping cells), leading to very overestimated modulus values. As the computation scheme applies only outer walls on the cell on the side of its umbrella, an insufficiently fine mesh on the opposite side can lead to this overstepping. This effect can only be avoided with certainty for all discretization lengths by applying each outer wall to every Voronoï cell, with a corresponding increase in computation time, and an inability to process non-convex shapes.

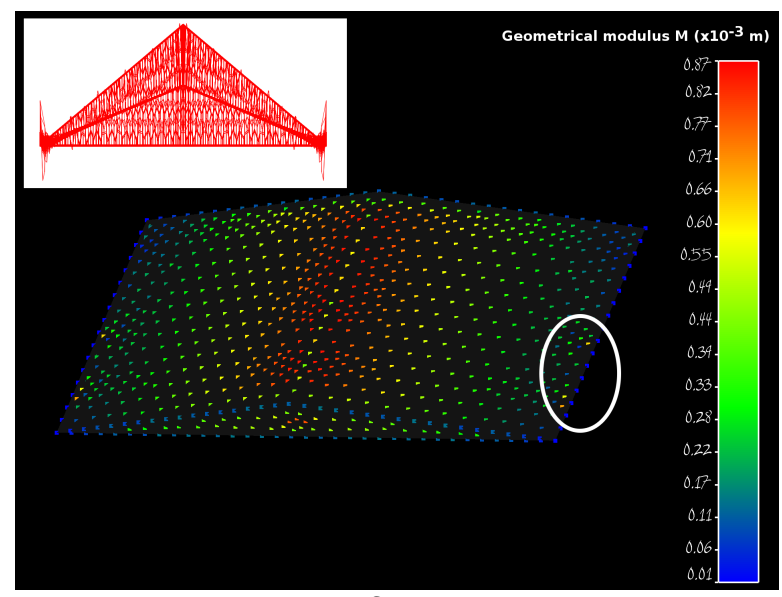

Figure 11: Example of out-stepping computed Voronoï cell centered on high geometrical modulus error vertices in a prism shape $(0.6$ $\mathrm{mm}$ discretization length)

\section{Analysis of complex (industrial) part:}

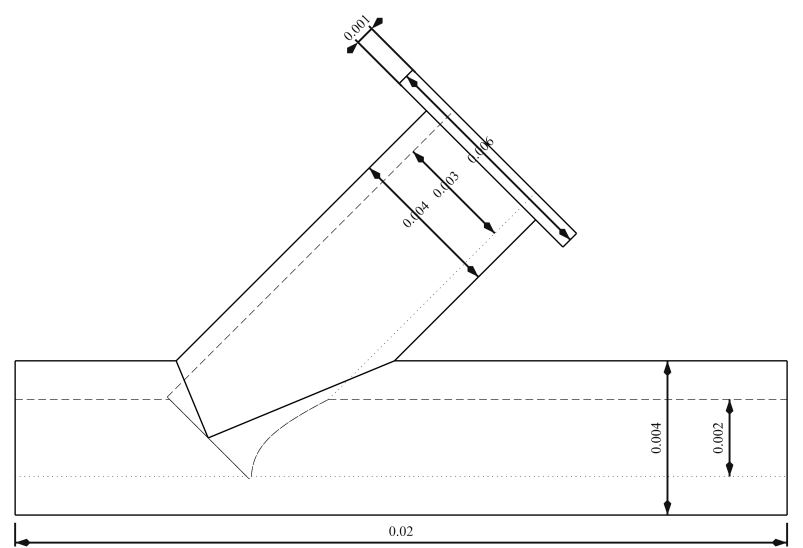

Figure 12: Connector part draft (dimensions in meters)

The shape studied is a connector between 2 tubes with one flat flange as seen in Fig. 12. These tubes have $20 \mathrm{~mm}$ outer radiuses, with one having $10 \mathrm{~mm}$ inner radius, and the other $15 \mathrm{~mm}$. Simple geometrical considerations give the theoretical values for isolated tubes geometrical moduli: $M=5$ $\mathrm{mm}$ and $\mathrm{M}=2.5 \mathrm{~mm}$. Due to shape complexity, geometrical modulus at the junction cannot be easily evaluated. Discretization of the solid surface has been carried out, with a maximum length of $\lambda=2.5$ $\mathrm{mm}$, leading to a mesh containing about 3600 vertices, which was subsequently exported to .stl format. Total computation time for geometrical modulus calculations of this file was 26 seconds. This duration is probably far longer than an optimized program would take, as the version of the algorithm used generates several intermediate (and unnecessary) files for control and study purpose. The modulus mapping obtained is shown in Fig. 13. The following observations can be carried out:

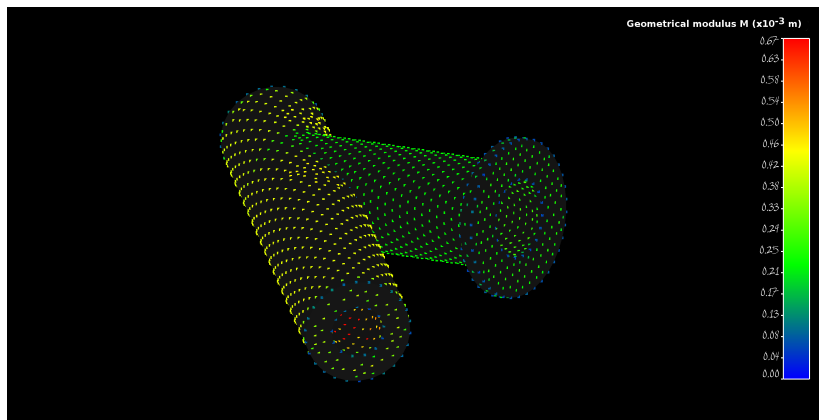

Figure 13: Modulus mapping of connector part

- $\quad$ rapidly cooling areas (edges of the shape) can easily be identified, contrary to traditional approach, where they are considered part of larger geometrical entities.

- modulus values computed far from the junction are more precise than given by the traditional approach. The Voronoï cells method automatically takes the curvature into account, leading to differing modulus values inside and outside of hollow cylinders. For example, instead of a global value of $2.5 \mathrm{~mm}$ for a whole cylinder, the surface should have a theoretical modulus of $2.3 \mathrm{~mm}$ outside and $2.8 \mathrm{~mm}$ inside, which corresponds to experimental results $(2 \cdot 2$ and 3.1 $\mathrm{mm}$ respectively).

- the modulus inside the thicker tube is less homogeneous than in other parts of the connector, which locally hides the curvatures effect; the variations, however, are under $10 \%$, which corresponds roughly to the shape approximation of a $10 \mathrm{~mm}$ curvature with a 2.5 $\mathrm{mm}$ discretization length. As such, a smaller discretization length can easily improve homogeneity and accuracy.

For comparison purpose, the same (discretized) shape was analyzed using finite elements method (using Elmer FEM solver), as well as its 
corresponding green-sand mold, with the starting hypothesis of liquid filled mold (simple cooling). Physical parameters used for the simulation are those of AISi12 alloy, given in Table 2 [Table 2 near here], and have been obtained from the following papers: [17 - 20]. Simulated cooling duration was $60 \mathrm{~s}$, to ensure solidification of the whole part (computation time is 8660s for 281000 elements). The main obstacle for comparisons is the difference in results types between the two methods: Voronoï cell approach gives an indicator of the time needed to solidify a volume cell (last point of the cell solidified), while FEM simulations gives local temperature evolution at vertices. In order to express these differing results in a comparable way, vertices data had to be converted to cell data. This was done by linking each vertex to the corresponding Voronoï cell, then considering the solidification time for the cell as the highest solidification time of vertices it contains. These data were then converted to geometrical modulus values using equation 1, with parameters carefully chosen to obtain a range of values comparable with Voronoï computation results (see Fig. 14). The main comparison results are following:

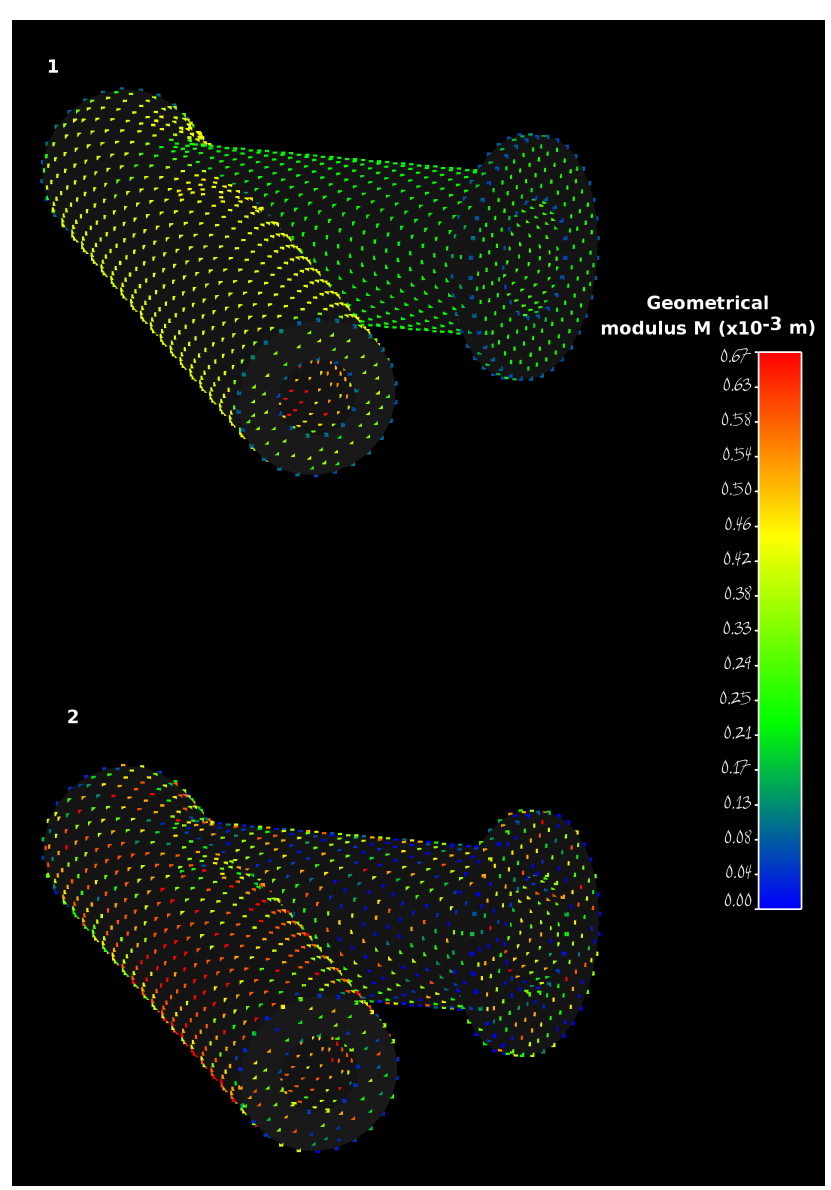

Figure 14: Comparison of geometrical modulus mapping obtained by (1) Voronoï cells method and (2) post-treated FEM simulation data, for connector part
- $\quad$ Post-treated FEM data show many aberrant points, as visible on Fig. 14-2. Such phenomenon seems more pronounced in thin components of the part. This effect is closely linked to the conversion from vertex data to cell data, as each cell contains an average of only 3 vertices, leading to errors in estimated solidification time by FEM (some cells have no vertex to estimate cooling duration, and others have vertices at non representative locations). Statistically, these errors are more probable in thinner areas, as the vertices density is constant throughout the part, and could be limited by the use of a very fine FEM mesh.

- Theoretical Voronoï cells cooling difference between the inside and outside of tubes was not easily evidenced in simulation results (Fig. 14-2) contrary to Voronoï approach (Fig. 14-1), which is most likely a consequence of the relatively coarse volume meshing (a few elements in the thickness of the part). However, an asymmetry of temperature distribution in tube thickness is visible in raw FEM temperature data (see Fig. 15), hinting that this effect indeed exists, but with a lesser magnitude than calculated by Voronoï cells approach. The difference can easily be explained by the first order diffusion hypothesis used in the Voronoï method, compared to reevaluated diffusion direction at each simulation step.

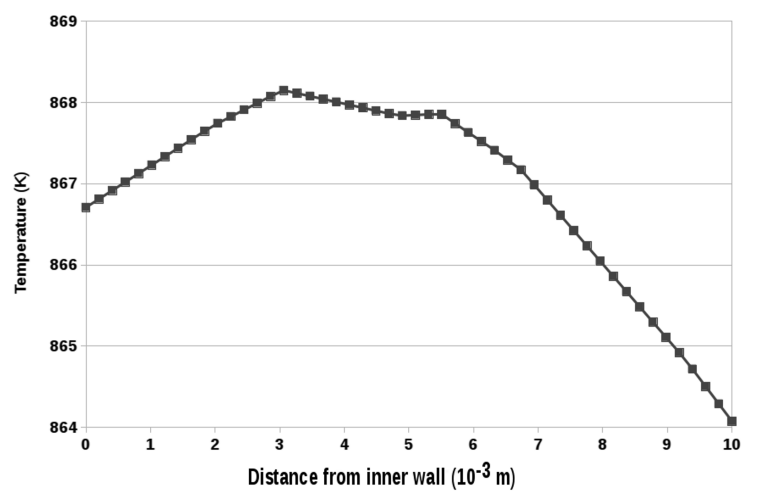

Figure 15: Temperature distribution in the thickness of tubular area from connector part

- Taking the preceding restrictions into account, Voronoï method seems to lead to geometrical modulus values comparable to estimations resulting from FEM computations. For macroscopic components of the casting (thin tube is around $0.25 \mathrm{~mm}$, and thick tube has a mean modulus of $0.5 \mathrm{~mm}$ for both internal and external faces, with a small asymmetry), these results show the determination of the same 
cooling sequence in the part using the two methods.

As a summary, the algorithm used to compute local geometrical modulus is far quicker than both traditional calculations and finite elements simulations. Its accuracy and data requirements are intermediate to the two other methods, with an increased precision in space compared to traditional approach, and lower accuracy (due to the simplified diffusion hypothesis involved) than in simulations.

\section{Conclusions:}

A local definition for geometrical modulus has been devised, based on the concept of Voronoï cells around a point of the surface of the casting, with properties in agreement with both pre-existing global expression of the modulus and initial (first order) thermal diffusion. Accurate approximations of continuous modulus mapping can be obtained by using discrete Voronoï cells constructions, centered on the vertices of a meshing of the casting (convergence is demonstrated in the case of plate shapes)

Numerical implementation of this concept has been achieved, using the Voro++ library as a basis for Voronoï cells computations, with some modifications to take non-convex geometries into account. The resulting program is very quick in comparison to numerical simulations (the complete process is about a few minutes in high density meshes), and takes standard .stl files as input, the output being simple text files containing vertices coordinates and their associated modulus.

Experimental computation tests have been carried out on simple shapes (plates, cylinders and wedges) to evidence the effects of various properties (mesh density, modulus gradients, curvatures) on the computed modulus accuracy. Results have instead shown the primary importance of mesh characteristics, to the point that other effects are completely concealed by it:

- mesh size / local modulus ratio sets the maximum possible amplitude for modulus errors

- the exact error (within these boundaries) happening on a given vertex is linked to local mesh configuration, such as mesh size discontinuity, or vertices misalignment on opposing faces
These elements give some clues to optimize the meshing of the casting shape:

- mesh size must be fine enough both to approximate accurately the shape of the casting, and to set an upper boundary for systematic errors in the geometrical modulus computations.

- $\quad$ a finer mesh size is needed in finer areas of the casting (which have a low modulus) to avoid proportionately larger errors. Experimental observations (not supported by theoretical work) seem to show that a discretization length of the mesh less than the underlying modulus value is enough to obtain an accurate modulus (less than $20 \%$ error) in a foundry context.

- mesh density variations should be progressive, in order to avoid errors linked with mesh size discontinuity.

Finally, comparison of this new approach with finite element simulation on a complex shape revealed that, although less precise than numerical simulations (due to the unidirectional thermal diffusion hypothesis inherent in the definition of geometrical modulus), the Voronoï cells method is both less demanding in physical data, and considerably quicker (around 300 times). As such, this approach does not constitute a suitable replacement for simulation, but instead would combine well with it, by using first several fast computation / casting designing cycles to optimize and refine casting shape (risers locations and sizes for example) and process, then using numerical simulation only once on the final shape to predict the part properties (grain size for example).

\section{Acknowledgements:}

We would like to thank M. Yves LICCIA, foundry teacher at Art et Métier ParisTech in Aix-enProvence (France), for his precious advice concerning traditional methods of casting design, and his vast experience in this field leading to interesting discussions.

Voronoï cells calculations in this study have been carried out using the Voro++ $\mathrm{C}++$ library, developed by Chris H. Rycroft. We thank him for developing this useful framework, and the very thorough and educational accompanying documentation. 


\section{Bibliographic references:}

1. Tiryakioglu M. A Comparative Study of Optimum Feeder Models for Castings. Int. J. Cast Met. Res. 2001;14:25-30.

2. Szucki M, Suchy JS. A voxelization based mesh generation algorithm for numerical models used in foundry engineering. Metall. Foundry Eng. 2012;38:43.

3. Pao W, Chen X, Chao L. Optimization of Insulation Padding for Directional Solidification. J. Appl. Sci. 2013;13:321-5.

4. United States Navy. Foundry Manual. Amsterdam: Fredonia Books; 1958.

5. Dey TK, Zhao W. Approximating the Medial Axis from the Voronoi Diagram with a Convergence Guarantee. Algorithmica. 2003;38:179-200.

6. Pao WKS, Ransing RS, Lewis RW, Lin C. A medial-axes-based interpolation method for solidification simulation. Finite Elem. Anal. Des. 2004;40:577-93.

7. Upadhya GK, Das S, Chandra U, Paul AJ. Modelling the investment casting process: a novel approach for view factor calculations and defect prediction. Appl. Math. Model. 1995;19:354-62.

8. Chvorinov N. Theory of solidification of castings. Giesserei. 1940;177-225.

9. Campbell J. Castings. Butterworth-Heinemann; 2003.

10. Jelínek $P$, Elbel $T$. Chvorinov's rule and determination of coefficient of heat accumulation of moulds with non-quartz base sands. 2010 [cited 2014 May 12]; Available from: http://core.kmi.open.ac.uk/display/18112102
11. Murat Tiryakioglu ET. Statistical investigation of the effects of the shape, size and superheat on solidification times of castings. AFS Trans. 1997;105:907-13.

12. Bern M, Eppstein D, Yao F. The Expected Extremes In A Delaunay Triangulation. 1991.

13. Arkin EM, Fernández Anta A, Mitchell JSB, Mosteiro MA. Probabilistic bounds on the length of a longest edge in Delaunay graphs of random points in d-dimensions. Comput. Geom. 2015;48:134-46.

14. Chris H. Rycroft. VORO++: A three-dimensional Voronoi cell library in $\mathrm{C}++$. Chaos Interdiscip. J. Nonlinear Sci. 2009;19:041111.

15. Chris H. Rycroft. Voro++ - A 3D Voronoi cell software library [Internet]. 2008 [cited 2014 Oct 21]. Available from: http://math.Ibl.gov/voro++/

16. Bajaj C, Dey T. Convex Decomposition of Polyhedra and Robustness. SIAM J. Comput. 1992;21:339-64.

17. Konopka Z, Lagiewka M, Zyska A. Evaluation of the heat transfer coefficient at the metal-mould interface during flow. Arch. Foundry Eng. 2007;7:101-4.

18. Krajewski PK, Suchy JS, Piwowarski G, Krajewski WK. High Temperature Thermal Properties of Bentonite Foundry Sand. Arch. Foundry Eng. 2015;15:47-50.

19. Pariona MM, Mossi AC. Numerical simulation of heat transfer during the solidification of pure iron in sand and mullite molds. J. Braz. Soc. Mech. Sci. Eng. 2005;27:399-406.

20. Sun H-C, Chao L-S. An Investigation into the Effective Heat Transfer Coefficient in the Casting of Aluminum in a Green-Sand Mold. Mater. Trans. 2009;50:1396-403. 
Tables :

Table 1: Summary of parameters studied on the frustum shape

\begin{tabular}{|c|c|c|c|c|}
\hline $\begin{array}{l}\text { Small face } \\
\text { radius }\left(\times 10^{-3} \mathrm{~m}\right)\end{array}$ & $\begin{array}{c}\text { Large face radius } \\
\quad\left(\times 10^{-3} \mathrm{~m}\right)\end{array}$ & $\begin{array}{c}\text { Nominal } \\
\text { discretization } \\
\text { length }\left(\times 10^{-3} \mathrm{~m}\right)\end{array}$ & $\begin{array}{c}\text { Effective mean } \\
\text { discretization length } \\
\left(\times 10^{-3} \mathrm{~m}-\text { small face }\right)\end{array}$ & $\begin{array}{c}\text { Effective } \\
\text { mean } \\
\text { discretization } \\
\text { length (x10- } \\
{ }^{3} \mathrm{~m}-\text { large } \\
\text { face) }\end{array}$ \\
\hline 5 & 5 & 0,5 & 0,48 & 0,48 \\
\hline 5 & 5 & 0,7 & 1,044 & 1,044 \\
\hline 5 & 5,1 & 0,5 & 0,48 & 0,499 \\
\hline 5 & 5,1 & 0,7 & 1,044 & 1,073 \\
\hline 5 & 5,2 & 0,5 & 0,48 & 0,516 \\
\hline 5 & 5,2 & 0,7 & 1,044 & 1,126 \\
\hline 5 & 5,3 & 0,5 & 0,48 & 0,538 \\
\hline 5 & 5,3 & 0,7 & 1,044 & 1,147 \\
\hline 5 & 5,4 & 0,5 & 0,48 & 0,556 \\
\hline 5 & 5,4 & 0,7 & 1,044 & 1,196 \\
\hline 5 & 5,5 & 0,5 & 0,48 & 0,574 \\
\hline 5 & 5,6 & 0,5 & 0,48 & 0,597 \\
\hline 5 & 5,7 & 0,5 & 0,48 & 0,617 \\
\hline 5 & 5,8 & 0,5 & 0,48 & 0,642 \\
\hline 5 & 5,9 & 0,5 & 0,48 & 0,659 \\
\hline 5 & 10 & 0,5 & 0,48 & 0,3187 \\
\hline
\end{tabular}


Table 2: Materials parameters and boundaries conditions used for finite elements simulation of connector part

\begin{tabular}{|c|c|c|}
\hline & AlSi12 & Green sand mold \\
\hline Density $\left(\mathrm{kg} \cdot \mathrm{m}^{-3}\right)$ & 2820 & 4500 \\
\hline Heat capacity $\left(\mathrm{J} \cdot \mathrm{kg}^{-1} \cdot \mathrm{K}^{-1}\right)$ & 970 & 0,53 \\
\hline Conductivity $\left({\left.\mathrm{W} \cdot \mathrm{m}^{-1} \cdot \mathrm{K}^{-1}\right)}^{\text {Initial temperature }(\mathrm{K})}\right.$ & 130 & 293 \\
\hline $\begin{array}{c}\text { Metal / sand heat transfer } \\
\text { coefficient }\left(\mathrm{W} \cdot \mathrm{m}^{-2} \cdot \mathrm{K}^{-1}\right)\end{array}$ & 900 & 1 \\
\hline Overheating $(\mathrm{K})$ & $50(900 \mathrm{~K})$ \\
\hline
\end{tabular}

